\title{
Article \\ A New Composite Material on the Base of Carbon Nanotubes and Boron Clusters B12 as the Base for High-Performance Supercapacitor Electrodes
}

\author{
Dmitry A. Kolosov ${ }^{1}$ and Olga E. Glukhova ${ }^{1,2, *(1)}$ \\ 1 Department of Physics, Saratov State University, Astrakhanskaya 83, 410012 Saratov, Russia; \\ kolosovda@bk.ru \\ 2 Laboratory of Biomedical Nanotechnology, I.M. Sechenov First Moscow State Medical University, Bolshaya \\ Pirogovskaya Street 2-4, 119991 Moscow, Russia \\ * Correspondence: glukhovaoe@info.sgu.ru
}

check for

updates

Citation: Kolosov, D.A.; Glukhova O.E. A New Composite Material on the Base of Carbon Nanotubes and Boron Clusters B12 as the Base for High-Performance Supercapacitor Electrodes. C 2021, 7, 26. https:// doi.org/10.3390/c7010026

Received: 28 January 2021

Accepted: 23 February 2021

Published: 25 February 2021

Publisher's Note: MDPI stays neutral with regard to jurisdictional claims in published maps and institutional affiliations.

Copyright: (c) 2021 by the authors. Licensee MDPI, Basel, Switzerland. This article is an open access article distributed under the terms and conditions of the Creative Commons Attribution (CC BY) license (https:// creativecommons.org/licenses/by/ $4.0 /)$.

\begin{abstract}
We explore the quantum capacitance, stability, and electronic properties of single-walled carbon nanotubes decorated with B12 icosahedral boron clusters by first-principle calculation methods implemented in the SIESTA code. After the optimization of the built supercells, the B12 clusters formed bonds with the walls of the carbon nanotubes and demonstrated metallic properties in all cases. The network of carbon nanotubes with its large area and branched surface is able to increase the capacity of the electric double-layer capacity, but the low quantum capacity of each nanotube in this network limits its application in supercapacitors. We found that the addition of boron clusters to both the outer and inner walls increased the quantum capacitance of carbon nanotubes. The calculation of the transmission function near the Fermi energy showed an increase in the conductivity of supercells. It was also found that an increase in the concentration of boron clusters in the structure led to a decrease in the heat of formation that positively affects the stability of supercells. The calculation of the specific charge density showed that with an increase in the boron concentration, the considered material demonstrated the properties of an asymmetric electrode.
\end{abstract}

Keywords: quantum capacitance; single-walled carbon nanotubes; supercapacitors; charge transport

\section{Introduction}

Supercapacitors (SC) are devices with high capacity, power density and long lifetime. They are designed for highly efficient electricity storage and occupy an intermediate position between dielectric capacitors and accumulators [1-5]. SC are also called as electric double-layer capacitors (EDLC), since the charge accumulation occurs at the interface between the electrode and the electrolyte. The electrical capacity of EDLC is much higher than that of traditional dielectric capacitors. As it is known, electrodes of conventional capacitors are made from a metal foil whose small area prevents an increase in the electrical capacity. However, at the present time, supercapacitors use various types of carbon materials with developed surface, for example, activated carbon, where at the electrodeelectrolyte interface of a large area electrostatic charge accumulation with reversible ion adsorption is possible [6]. In terms of power density, EDLC occupy a leading position among electrochemical sources, but their energy density is still significantly lower than that of batteries $[7,8]$. Materials with high electrical conductivity and developed surface for forming a double electrical layer can increase the efficiency and capacity of existing EDLC. Currently, promising materials in this area are carbon nanotubes (CNTs) and graphene [9-12]. However, the formation of a double layer is not a key factor in increasing the electrical capacity of the EDLC. It was noted that the low quantum capacitance (QC) of 
carbon materials used as electrodes significantly affected the total EDLC capacity $[13,14]$. This relationship is described by the equation:

$$
\frac{1}{\mathrm{C}_{\text {Total }}}=\frac{1}{\mathrm{C}_{\mathrm{D}}}+\frac{1}{\mathrm{C}_{\mathrm{Q}}}
$$

where $\mathrm{C}_{\text {Total }}$ is the total capacity of EDLC, $\mathrm{C}_{\mathrm{D}}$-capacity of a double layer, $\mathrm{C}_{\mathrm{Q}-\mathrm{QC}}$. It follows from Equation (1) that the low QC neutralizes the contribution of the double electric layer to the total EDLC capacitance. The question arises: How to increase the QC? A number of theoretical works demonstrate that an increase in the $\mathrm{QC}$ of carbon materials is possible by the adsorption of metal atoms and some nonmetals on their surface or by creating vacancy defects [15-17]. These theoretical results are confirmed by experimental works $[18,19]$, which show that modified CNTs have a much larger capacity in comparison to pure CNTs. CNTs can be modified by the adsorption of various atoms and atomic clusters on their walls. One of the most successful CNT modifications is functionalization with boron-containing compounds. For example, in [20], it was shown that the EDLC electrode based on MnO2-CNT-Boron demonstrated the specific total capacity of $1544 \mathrm{~F} / \mathrm{g}$ after 5000 charge/discharge cycles. The electrode material on the base of multi-walled CNTs doped with boron nitride has the high specific power density of $220 \mathrm{~W} / \mathrm{kg}$ [21]. The supercapacitors based on CNT and boron nitride are recognized as one of the most effective: its electrode retains $98 \%$ of its original total capacity after 10,000 charge/discharge cycles [22].

Thus, numerous modern scientific studies, both experimental and theoretical, prove the relevance and prospects of EDLC electrode materials. At the same time, increasing the efficiency of these materials is really possible due to preliminary research of ways to increase the QC by changing the topology and morphology of the applied materials. An increase in the QC will inevitably lead to an increase in the total capacity of the entire EDLC electrode.

In this work, we identify (I) the dependence of $\mathrm{QC}$ of the electrode material based on CNT/B12 on the mass concentration of boron, (II) the regularities of the charge transfer between CNT and B12 clusters, and (III) the regularities of quantum electron transport in $\mathrm{CNT} / \mathrm{B} 12$ material.

\section{Materials and Methods}

The search for the ground structure as well as calculations of the electronic structure and energy parameters were performed by the density functional theory (DFT) method using the SIESTA 4.1 software $[23,24]$ with application of the double- $\zeta$ plus polarization (DZP) basis set. For the expression of the exchange-correlation energy between interacting electrons, the generalized gradient approximation (GGA) with Perdew-Burke-Ernzerhof (PBE) parameterization was used [25]. The total energy minimization was performed with the application of Hellmann-Feynman forces including Pulay-like corrections and using the modified Broyden algorithm [26]. The variable parameters were atoms coordinates and translation vector lengths. The optimization process was finished when the force acting on each atom became less than $0.025 \mathrm{eV} / \AA$. The temperature in the calculation process was fixed at $300 \mathrm{~K}$. A mesh cutoff of $350 \mathrm{Ry}$ was used for the computation of the electron densities and potentials. The Monkhorst-Pack method with a $1 \times 1 \times 15$ grid was used to sample k-points in the Brillouin zone. The transport properties were calculated using the nonequilibrium Green's Function (NEGF) formalism in the TranSIESTA package included in the SIESTA code.

The $Q_{C} C_{Q}$ was calculated by the formula $[27,28]$ :

$$
\mathrm{C}_{\mathrm{Q}}(\mathrm{V})=\frac{1}{\mathrm{mV}} \int_{0}^{\mathrm{V}} \mathrm{eD}\left(\mathrm{E}_{\mathrm{F}}-\mathrm{eV}\right) \mathrm{dV}
$$


where $\mathrm{m}$ is mass of an object, $\mathrm{V}$ is bias calculated as the change of the Fermi level $\mathrm{E}_{\mathrm{F}}$ with a change in the object's charge, e is elementary charge, and D is density of states (DOS) under applied bias.

\section{Results}

\subsection{Atomistic Models}

The atomistic model of the EDLC electrode material was a CNT (21.0) with a length of $11.36 \AA$ decorated with boron clusters B12. The translation vector was directed along the Y-axis (along the axis of the tube) and was $12.94 \AA$. The diameter of the CNT was $16.46 \AA$. The choice of such a CNT diameter was because tubes of this diameter are most often synthesized [29] and demonstrate a metallic type of conductivity. The clusters used for the modification of nanotubes had an icosahedral shape and contained 12 boron atoms in its structure. At the moment, electrodes based on boron clusters are being actively studied. For example, in [30], the electrode material based on B12 clusters and tungsten oxide showed high cycling, retaining $96 \%$ of its original capacity. Figure $1 \mathrm{a}-\mathrm{f}$ show the optimized supercells of the EDLC electrode material. For convenience, the structure with a single boron cluster is shown at different angles in Figure 1a,b.
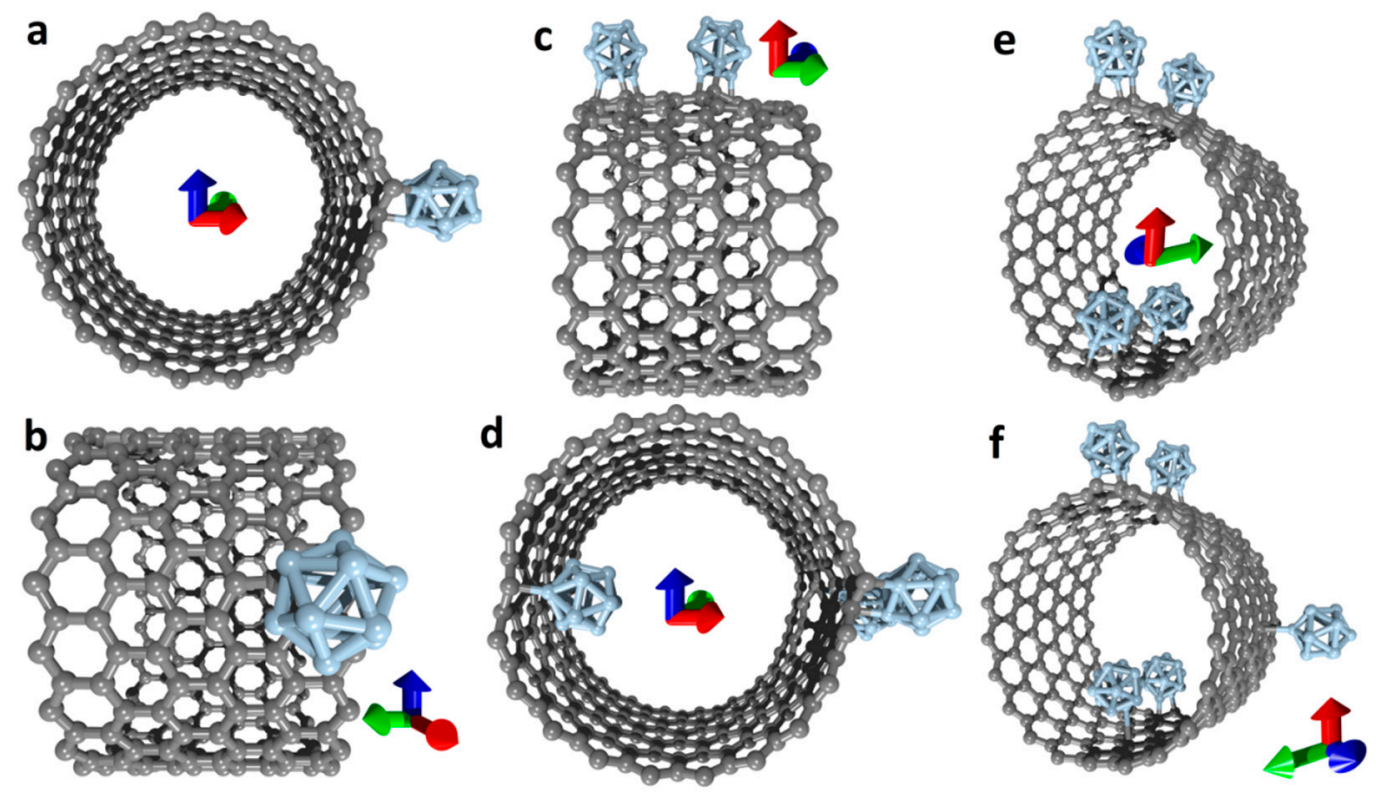

Figure 1. The supercells of the boron-modified
(d) CNT@B12x3; (e) CNT@B12x4; (f) CNT@B12x5.

As can be seen from Figure 1, five models of the supercells with different numbers of boron clusters and constant nanotube length were built. The boron and carbon atoms formed bonds. The clusters were located both outside and inside of the tube. The clusters were connected sequentially one by one. For the tube of this length, the concentration of boron clusters was not the maximum possible. The mass fraction of boron ranged from 4.12 to $17.68 \%$. The bond length between the boron and carbon atoms on the inner wall of the CNT was $1.70 \AA$. On the outer wall, the bond lengths were already in the range $1.665-1.674 \AA$ A. It should also be noted that the formation energy between the cluster and the tube from the outside was more favorable.

\subsection{Electronic and Transport Properties}

To estimate the effect of boron clusters on the electronic structure of CNTs, the charge transfer distribution (Figure 2) and DOS (Figure 3) were calculated. As a result of the charge redistribution, in all cases, the tube reported the charge to the boron clusters. At the same time, the clusters inside the tube also lose charge, while the external ones are 
received. The red color shows the atoms with a deficit of electronic charge. Figure 2e shows the numbering of the clusters and the corresponding charges. It is clearly seen that the internal clusters have lost a little charge, and the external ones have received a little charge. Moreover, the close-sitting clusters No. 1 and 2 together accepted noticeably more charge. So, the cluster landing density plays an important role in charge transfer.
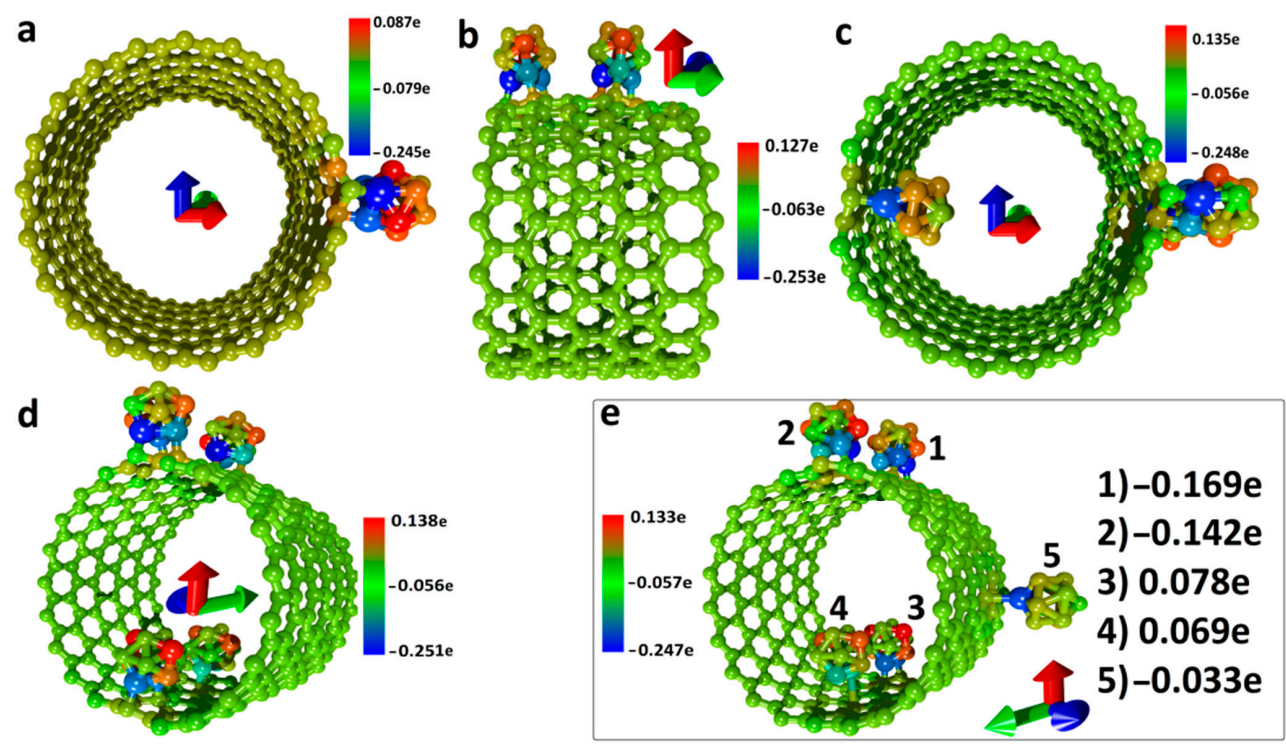

Figure 2. The electron density redistribution: (a) CNT@B12x1; (b) CNT@B12x2; (c) CNT@B12x3; (d) CNT@B12x4; (e) CNT@B12x5.

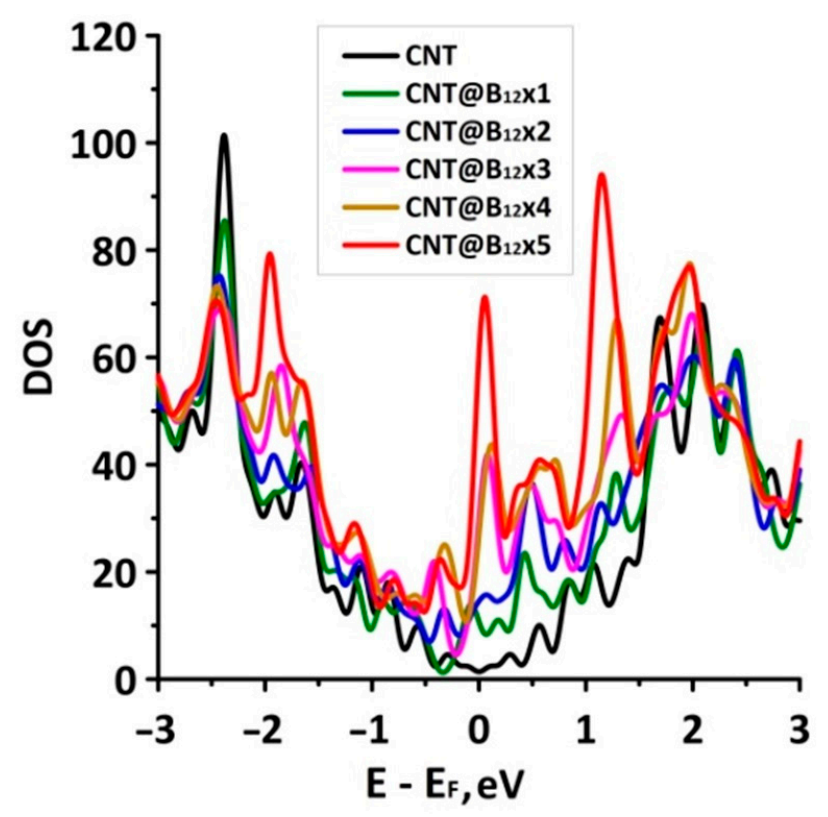

Figure 3. The DOS graph for the pure carbon nanotubes (CNTs) and for CNTs decorated with different numbers of boron clusters.

In Figure 3, the black line corresponds to a pure $\mathrm{CNT}^{\prime}$ s DOS curve ( $\mathrm{E}_{\mathrm{F}}-$ Fermi level). Modification of a CNT by boron clusters increased the number of electronic states near the Fermi level, and the profile of the curves became asymmetric. Each addition of a boron cluster increased the intensity of DOS peaks. It can be concluded that boron clusters increased the conductivity of this material, which is very important for its application in the EDLC electrode material. 
Next, we calculated the specific QC (CQ) of the studied structures at the different voltage values (Figure 4). Unlike the pure CNT (black curve), the QC of the boron-modified tubes had local maxima near zero volts. With a negative voltage shift in the range from -2 to $-3 \mathrm{~V}$, the change in $\mathrm{QC}$ was almost the same for each model. However, for the positive voltage shift, the growth of QC occurred abruptly, especially in the range from 0 to $1 \mathrm{~V}$. This was caused by the increase in DOS near the Fermi energy. The maximum value of QC was $\approx 850 \mathrm{~F} / \mathrm{g}$ at a bias voltage of $0.1 \mathrm{~V}$. It was achieved in the case of modification by the maximum number of boron clusters. The specific charge density Q_SC of the modified CNTs was calculated by the formula [31]:

$$
\mathrm{Q}_{\mathrm{SC}}=\int_{0}^{\mathrm{V}} \mathrm{C}_{\mathrm{Q}}(\mathrm{V}) \mathrm{dV}
$$

where $C_{Q}(V)$ is $Q C$ under the local potential of the electrode V. Graphs are shown in Figure 5. The branch of pure CNT (black) was zero-symmetric. With the addition of boron, the symmetry immediately disappeared, especially for the 4 and 5 boron clusters. The asymmetry of the branches allows us to conclude that this electrode material belongs to the so-called hybrid of a supercapacitor and a battery that can provide both the advantages of a battery (high energy density) and a capacitor (high power density) [32].

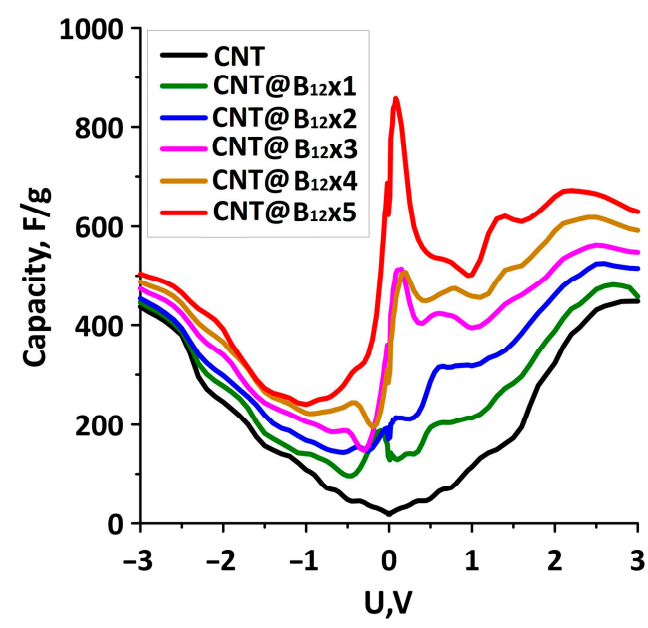

Figure 4. The quantum capacitance (QC) graph for the pure $\mathrm{CNT}$ and for the $\mathrm{CNTs}$ decorated with different numbers of boron clusters.

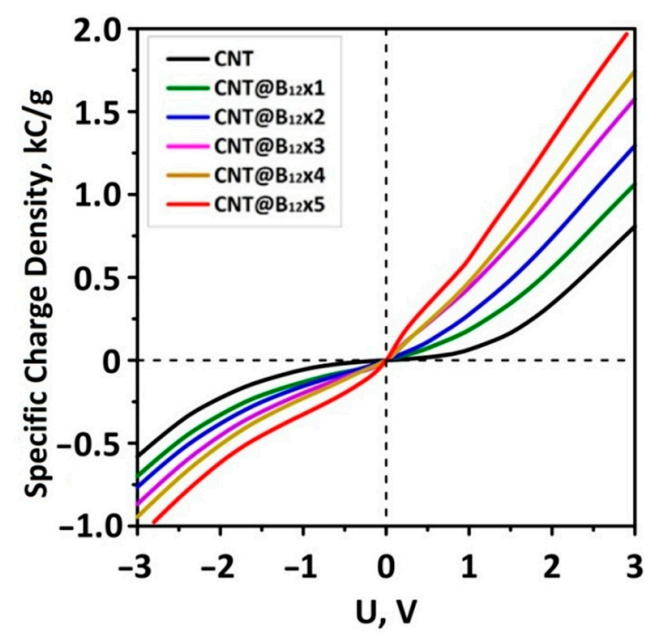

Figure 5. The specific charge density graph for the pure CNT and for the CNTs decorated with different numbers of boron clusters. 
At the final stage, for the CNT/B12 supercells, we calculated the transmission function near the Fermi level followed by the calculation of the specific resistivity. As it is known, the EDLC electrode material must have a low specific resistivity to create large discharge and charge currents. In our work, we do not take into account all the parameters of the equivalent series resistance (ESR) of the EDLC material, but the estimation of the resistivity of the electrode material is extremely important, since it is one of the terms for calculating the ESR of the EDLC material [33]. Figure 6 shows the profiles of the transmission functions for the modified CNT. The addition of boron clusters significantly changed the transmission function of the EDLC electrode material models, increasing the number of conduction quanta. At the Fermi level, the CNT@B12x5 model had five conduction quanta, but at the same time, the CNT@B12x1 models had only one quantum. It should be noted that all the profiles of the transmission function contained gaps, but for the pure tube, the gap was located directly near the Fermi level, while for the modified tubes, the gap was shifted from the Fermi level. This result was expected due to the large DOS values at the Fermi level in the modified CNTs. Table 1 below shows the numerical results of the study.

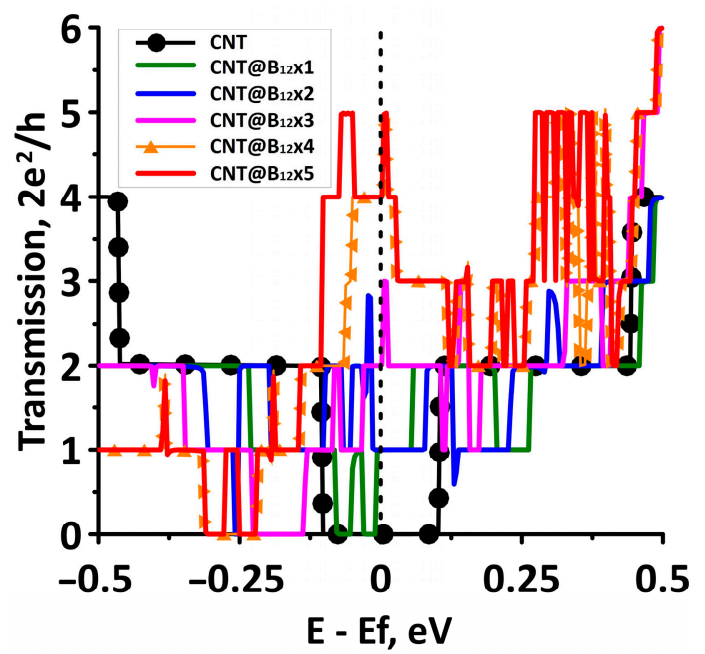

Figure 6. Transmission functions for the pure CNT and for the CNTs decorated with different numbers of boron clusters.

Table 1. The values of specific quantum resistance under zero voltage and specific resistance for pure CNT and for CNTs decorated with different numbers of boron clusters.

\begin{tabular}{|c|c|c|c|c|c|c|c|}
\hline Model & $\begin{array}{c}\text { Number of } \\
\text { Carbon } \\
\text { (Atoms) }\end{array}$ & $\begin{array}{c}\text { Number of } \\
\text { Boron (Atoms) }\end{array}$ & $\begin{array}{l}\text { Mass Ration } \\
\text { of the Boron, } \\
\text { (wt \%) }\end{array}$ & $\begin{array}{c}\text { Heat of } \\
\text { Formation per } \\
\text { Atoms, }(\mathrm{eV})\end{array}$ & $E_{F},(e V)$ & $\begin{array}{l}\text { Specific Quantum } \\
\text { Capacity under } \\
\text { Zero Voltage, (F/g) }\end{array}$ & $\begin{array}{c}\text { Specific } \\
\text { Resistance, } \\
(\mu \mathrm{Ohm} \cdot \mathrm{m})\end{array}$ \\
\hline CNT & 252 & 0 & 0 & - & -4.768 & 20 & 237.2 \\
\hline 1 & 252 & 12 & 4.12 & -0.01498 & -4.818 & 120 & 19.91 \\
\hline 2 & 252 & 24 & 7.906 & -0.02004 & -4.819 & 200 & 12.13 \\
\hline 3 & 252 & 36 & 11.42 & -0.02301 & -4.856 & 502 & 8.78 \\
\hline 4 & 252 & 48 & 14.66 & -0.02676 & -4.887 & 500 & 4.52 \\
\hline 5 & 252 & 60 & 17.68 & -0.02715 & -4.909 & 850 & 4.14 \\
\hline
\end{tabular}

The results of numerical simulation are in qualitative agreement with the results of the experimental work. Indeed, decorating CNTs with boron-containing compounds leads to an increase in the total capacity of the electrodes, which is confirmed by experimental studies [22,34]. It was also experimentally shown in [21] that boron-modified CNTs have lower ohmic resistance, charge transfer resistance, and resistance to ion transfer in the electrolyte than pure CNTs. These differences suggest that boron-modified CNTs have 
better charge transfer capabilities, allowing ions to move more easily. In addition, such materials have a higher adsorption capacity. In addition, using $\mathrm{X}$-ray energy spectrum analysis (EDS), X-ray photoelectron spectroscopy (XPS), as well as a specific surface area and porosimetry analyzer Brunauer-Emmett-Teller (BET) analyzer, it was found that boron atoms formed bonds with each other, which confirms the energy electronic stability of the material on based on CNT and boron.

\section{Conclusions}

In the present paper, the objects of the research were models of single-walled CNTs decorated with icosahedral B12 boron clusters. On the base of ab initio calculations performed in the SIESTA software, the energy stability as well as the electronic and the conductive properties of CNTs modified by B12 boron clusters were explored. We found that the addition of boron clusters to both the outer and inner walls of the CNT increased the quantum capacity of carbon nanotubes. The clusters located inside the CNTs reported their charge to the CNTs. The calculation of the transmission function revealed a sharp increase in the conductivity of the modified tubes. The resistivity decreased from 237 to $4.14 \mu \mathrm{Ohm} \cdot \mathrm{m}$ in comparison to the pure CNT. An increase in the concentration of boron clusters in the electrode material reduced its heat of formation. The calculation of the specific charge density demonstrated the asymmetry relative to the positive and negative bias. The obtained results expand the application of electrode material for asymmetric types of EDLC.

Author Contributions: Conceptualization, O.E.G. and D.A.K.; methodology, O.E.G.; investigation, O.E.G. and D.A.K.; writing—original draft preparation, D.A.K.; writing—review and editing, O.E.G.; supervision, O.E.G.; funding acquisition, D.A.K. All authors have read and agreed to the published version of the manuscript.

Funding: This research was funded by the RFBR, grant number 19-32-90160.

Institutional Review Board Statement: Not applicable.

Informed Consent Statement: Not applicable.

Data Availability Statement: Not applicable.

Conflicts of Interest: The authors declare no conflict of interest.

\section{References}

1. Poonam; Sharma, K.; Arora, A.; Tripathi, S.K. Review of supercapacitors: Materials and devices. J. Energy Storage 2019, 21, 801-825.

2. Zheng, S.; Wu, Z.-S.; Wang, S.; Xiao, H.; Zhou, F.; Sun, C.; Bao, X.; Cheng, H.-M. Graphene-based materials for high-voltage and high-energy asymmetric supercapacitors. Energy Storage Mater. 2017, 6, 70-97. [CrossRef]

3. Kondrat, S.; Kornyshev, A.A. Pressing a spring: What does it take to maximize the energy storage in nanoporous supercapacitors? Nanoscale Horiz. 2015, 1, 45-52. [CrossRef]

4. Zhou, H.; Liu, G.; Liu, J.; Wang, Y.; Ai, Q.; Huang, J.; Yuan, Z.; Tan, L.; Chen, Y. Effective Network Formation of PEDOT by in-situ Polymerization Using Novel Organic Template and Nanocomposite Supercapacitor. Electrochim. Acta 2017, 247, 871-879. [CrossRef]

5. Xu, L.; Jia, M.; Li, Y.; Zhang, S.; Jin, X. Design and synthesis of graphene/activated carbon/polypyrrole flexible supercapacitor electrodes. RSC Adv. 2017, 7, 31342-31351. [CrossRef]

6. Conway, B.E. Electrochemical Supercapacitors; Springer: Boston, MA, USA, 1999; pp. 1-698.

7. Majumdar, D.; Mandal, M.; Bhattacharya, S.K. Journey from supercapacitors to supercapatteries: Recent advancements in electrochemical energy storage systems. Emergent Mater. 2020, 3, 347-367. [CrossRef]

8. Xiao, Z.; Wang, S.; Yan, X.; Liu, C.; Zhao, X.; Yang, X. Integrating Fast Potential-Fringe Battery Reactions for High-Voltage Battery-Supercapacitor Hybrid Energy Storage. Batter. Supercaps 2019, 2, 766-773. [CrossRef]

9. Lu, Z.; Raad, R.; Safaei, F.; Xi, J.; Liu, Z.; Foroughi, J. Carbon Nanotube Based Fiber Supercapacitor as Wearable Energy Storage. Front. Mater. 2019, 6, 138. [CrossRef]

10. Liang, X.; Zhao, L.; Wang, Q.; Ma, Y.; Zhang, D. Dynamic stretchable and self-healable supercapacitor with CNT/graphene/PANI composite film. Nanoscale 2018, 10, 22329. [CrossRef]

11. Chinnasa, P.; Ponhan, W.; Choawunklang, W. Modeling and simulation of a LaCoO3 Nanofibers /CNT electrode for supercapacitor application. J. Phys. Conf. Ser. 2019, 1380, 012101. [CrossRef] 
12. Avasthi, P.; Kumar, A. Balakrishnan, Aligned CNT Forests on Stainless Steel Mesh for Flexible Supercapacitor Electrode with High Capacitance and Power Density. Acs Appl. Nano Mater. 2019, 2, 1484-1495. [CrossRef]

13. Paek, E.; Pak, A.J.; Hwang, G.S. A computational study of the interfacial structure and capacitance of graphene in [BMIM][PF6] ionic liquid. J. Electrochem. Soc. 2013, 160, A1-A10. [CrossRef]

14. Paek, E.; Pak, A.J.; Kweon, K.E.; Hwang, G.S. On the origin of the enhanced supercapacitor performance of nitrogen-doped graphene. J. Phys. Chem. C 2013, 117, 5610-5616. [CrossRef]

15. Hirunsit, P.; Liangruksa, M.; Khanchaitit, P. Electronic structures and quantum capacitance of monolayer and multilayer graphenes influenced by Al, B, N and P doping, and monovacancy: Theoretical study. Carbon 2016, 108, 7-20. [CrossRef]

16. Chen, L.; Li, X.; Ma, C.; Wang, M.; Zhou, J. Interaction and quantum capacitance of nitrogen/sulfur co-doped graphene: A theoretical calculation. J. Phys. Chem. C 2017, 121, 18344-18350. [CrossRef]

17. Sun, P.; Wang, R.; Wang, Q.; Wang, H.; Wang, X. Uniform MoS2 Nanolayer with Sulfur Vacancy on Carbon Nanotube Networks as Binder-free Electrodes for Asymmetrical supercapacitor. Appl. Surf. Sci. 2019, 475, 793-802. [CrossRef]

18. Miao, J.; Dong, X.; Xu, Y.; Zhai, Z.; Zhang, L.; Ren, B.; Liu, Z. Preparation and electrochemical performance of 1,4-naphthaquinonemodified carbon nanotubes as a supercapacitor material. Org. Electron. 2019, 73, 304-310. [CrossRef]

19. Wang, X.; Wu, D.; Song, X.; Du, W.; Zhao, X.; Zhang, D. Review on Carbon/Polyaniline Hybrids: Design and Synthesis for Supercapacitor. Molecules 2019, 24, 2263. [CrossRef]

20. Jain, R.; Wadekar, P.H.; Khose, R.V.; Pethsangave, D.A.; Some, S. MnO2@Polyaniline-CNT-boron-doped graphene as a freestanding binder-free electrode material for supercapacitor. J. Mater. Sci. Mater. Electron. 2020, 31, 8385-8393. [CrossRef]

21. Hao, H.; Xiaogang, S.; Wei, C.; Jie, W.; Xu, L.; Yapan, H.; Chengcheng, W.; Guodong, L. Electrochemical properties of supercapacitors using boron nitrogen double doped carbon nanotubes as conductive additive. Nano 2019, 14, 1950080.

22. Maity, C.K.; Sahoo, S.; Verma, K.; Behera, A.K.; Nayak, G.C. Facile functionalization of boron nitride (BN) for the development of high-performance asymmetric supercapacitors. New J. Chem. 2020, 44, 8106-8119. [CrossRef]

23. Ordejón, P.; Artecho, E.; Soler, J.M. Self-consistent order-N density-functional calculations for very large systems. Phys. Rev. B 1996, 53, 10441. [CrossRef]

24. Soler, J.M.; Artecho, E.; Gale, J.D.; Garcýa, A.; Junqera, J.; Ordejón, P.; Sanchez-Portal, D. The SIESTA method for ab initio order-N materials simulation. J. Phys. Condens. Matter 2002, 14, 2745. [CrossRef]

25. Perdew, J.P.; Burke, K.; Ernzerhof, M. Generalized Gradient Approximation Made Simple. Phys. Rev. Lett. 1996, 77, 3865. [CrossRef] [PubMed]

26. Johnson, D.D. Modified Broyden's method for accelerating convergence in self-consistent calculations. Phys. Rev. Lett. 1988, 38, 12807. [CrossRef] [PubMed]

27. Shunaev, V.V.; Ushakov, A.V.; Glukhova, O.E. Increase of gamma-Fe2O3/CNT composite quantum capacitance by structural design for performance optimization of electrode materials. Int. J. Quantum Chem. 2020, 120, e26165. [CrossRef]

28. Mousavi-Khoshdel, S.M.; Jahanbakhsh-bonab, P.; Targholi, E. Structural, electronic properties, and quantum capacitance of B, N and P-doped armchair carbon nanotubes. Phys. Lett. A 2016, 380, 3378-3383. [CrossRef]

29. Aguilar, Z.P. Types of Nanomaterials and Corresponding Methods of Synthesis; Elsevier: Amsterdam, The Netherlands, 2013 ; pp. 33-82.

30. Jung, D.; Muni, M.; Marin, G.; Ramachandran, R.; El-Kady, M.F.; Balandin, T.; Kaner, R.B.; Spokoyny, A.M. Enhancing cycling stability of tungsten oxide supercapacitor electrodes via a boron cluster-based molecular cross-linking approach. J. Mater. Chem. A 2020, 8, 18015-18023. [CrossRef]

31. Xu, Q.; Yang, G.; Fan, X.; Zheng, W. Improving the Quantum Capacitance of Graphene-Based Supercapacitors by the Doping and Co-Doping: First-Principles Calculations. ACS Omega 2019, 4, 13209-13217. [CrossRef] [PubMed]

32. Lai, C.; Wang, S.; Cheng, L.; Wang, Y.; Fu, L.; Sun, Y.; Lin, B. High-Performance Asymmetric Supercapacitors of Advanced Double Ion-buffering Reservoirs Based on battery-type Hierarchical Flower-like Co3O4-GC Microspheres and 3D Holey Graphene Aerogels. Electrochim. Acta 2020, 4, 13209-13217. [CrossRef]

33. Dolah, B.N.M.; Othman, M.A.R.; Deraman, M.; Basri, N.H.; Farma, R.; Talib, I.A.; Ishak, M.M. Supercapacitor Electrodes from Activated Carbon Monoliths and Carbon Nanotubes. J. Phys. Conf. Ser. 2013, 431, 012015. [CrossRef]

34. Maity, C.K.; Hatui, G.; Sahoo, S.; Saren, P.; Nayak, G.C. Boron Nitride based Ternary Nanocomposites with Different Carbonaceous Materials Decorated by Polyaniline for Supercapacitor Application. ChemistrySelect 2019, 4, 3672-3680. [CrossRef] 